\title{
Spatial revolute joints with clearances for dynamic analysis of multi-body systems
}

\author{
P Flores $^{1 *}$, J Ambrósio ${ }^{2}$, J C P Claro ${ }^{1}$, and H M Lankarani ${ }^{3}$ \\ ${ }^{1}$ Departamento de Engenharia Mecânica, Universidade do Minho, Guimarães, Portugal \\ ${ }^{2}$ Instituto de Engenharia Mecânica (IDMEC), Instituto Superior Técnico, Lisboa, Portugal \\ ${ }^{3}$ Mechanical Engineering Department, Wichita State University, Kansas, USA
}

The manuscript was received on 23 March 2006 and was accepted after revision for publication on 18 August 2006.

DOI: $10.1243 / 1464419 J M B D 70$

\begin{abstract}
This paper presents a new technique for assessing the influence that clearance of spatial joints has on the kinematics and dynamics of multi-body systems. The technique is developed for the revolute joint in which the basic elements are the journal and bearing. Under certain working conditions, these two mechanical elements collide with each other being the contact treated as coupled forces. The forces between each pair of contacting bodies are equal and opposite, and the magnitude and direction are calculated based on the positions and velocities of the bodies, as well as on the geometry of the joint. A continuous contact force model is used to evaluate the contact-impact force. The proposed methodology is developed in the framework of the multi-body systems formulation; therefore, the forces developed during the contact between the joint elements are introduced into the systems' equations of motion as external forces. A double pendulum is used as a numerical example to show the application of the proposed methodology, being the efficiency of the developed methodologies discussed in the process of the results obtained.
\end{abstract}

Keywords: joint clearances, spatial multi-body systems, contact forces, dynamics

\section{INTRODUCTION}

A mechanical joint, in any industrial machine, is designed to allow relative motion between the connected components. Owing to the manufacturing tolerances, wear, or material deformation, these joints are imperfect and have clearances. These clearances modify the dynamic response of the system, justify the deviations between the numerical predictions and the experimental measurements, and eventually lead to important deviations between the projected behaviour of the mechanisms and their real outcome. The attenuation of the impact response and of the vibration characteristics in industrial machines is obtained by including, in their design, a selection of joint clearances.

The problem of the dynamic behaviour of planar multi-body systems with joint clearances has been

\footnotetext{
* Corresponding Author: Departmento de Engenharia Mecanica, Universidade do Minho, Campus de Azurem, Guimaraes 480058, Portugal.email: pflores@dem.uminho.pt
}

studied in the past $[\mathbf{1}-\mathbf{6}]$. The utility of the methodologies developed is somewhat restricted because they are not valid for spatial multi-body systems, such as vehicle models, car suspensions, and robotic manipulators, where the system motion is not limited to be planar. In fact, even planar systems may exhibit out-of-plane motion due to misalignments, thus justifying the development of mathematical models to assess the influence of the joint clearances in spatial multi-body systems.

The typical configuration of a spatial revolute joint with clearance is schematically illustrated in Fig. 1. The pair of elements in a spatial revolute joint clearance is a cylindrical hole, the bearing, and a cylindrical pin, the journal. The clearance in a realistic connection is much smaller than the length of the two cylinders and of the nominal radius of the elements connected by the joint.

In the model presented here, the mechanical bodies connected by the joint are modelled as colliding rigid bodies, and, consequently, contact-impact forces control the dynamics of the joint. In this 


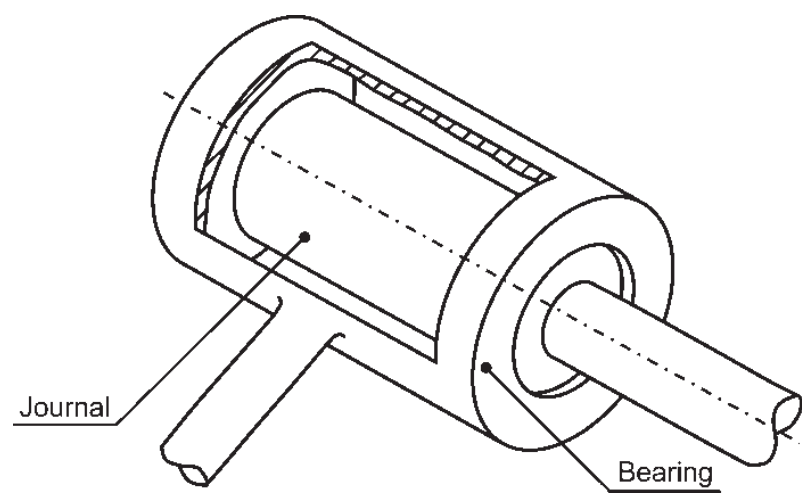

Fig. 1 Typical spatial revolute joint with clearance

methodology, the contact force model with hysteric damping is used to evaluate the time evolution of the normal contact forces resulting from the interpenetration between the journal and the bearing. For this purpose, the mechanical elements are considered as two rigid bodies in contact that penetrate into each other, without deforming. The normal contact force depends on this pseudo-penetration, according to the continuous force model proposed by Lankarani and Nikravesh [7]. Thus, it is clear that the spatial revolute joint with clearance does not impose kinematic constraints to the system, but imposes some force restrictions, limiting the journal movement within the bearing limits. This force model, which is function of the bodies' relative motion and of the internal geometry of the joint, leads to the contact forces that are introduced in the system's equations of motion.

The model for the spatial revolute joint clearances requires a large amount of mathematical manipulations, as there are different possible paths of motion for the journal when the clearance is present. Four different types of journal motions inside the bearing are considered in the present work, namely:

(a) free flight motion where there is no contact between the two elements;

(b) the journal contacts with the bearing wall at a point;

(c) the journal and the bearing contact with each other at a line;

(d) two contact points between the journal and bearing wall, but in opposite sides.

These four possibilities are illustrated in Fig. 2. The joint's behaviour is the function of these four scenarios which depend on the dynamic system's configuration.

In a non-contact situation, no forces are introduced into the system, because the journal moves freely inside the bearing boundaries until it reaches the bearing wall. When the journal and bearing are in contact with each other, local deformations take

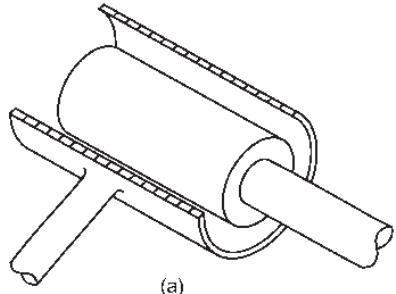

(a)

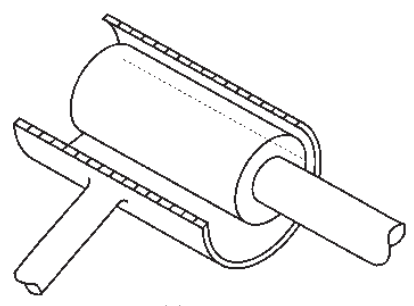

(c)

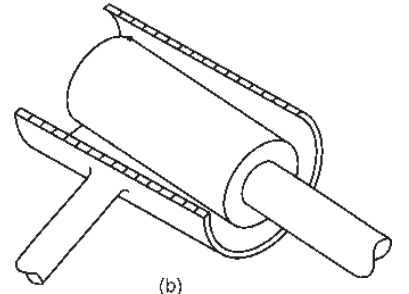

(b)

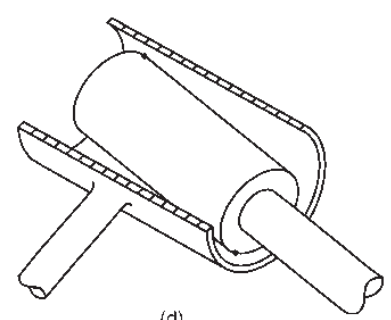

(d)
Fig. 2 Four different possible scenarios for the journal motion relative to the bearing: (a) no contact, (b) one point contact, (c) line contact, and (d) two contact points in opposite sides

place at the contact area and, consequently, contact-impact forces characterize the interaction between the bodies. By evaluating the variation of the contact forces during the contact period, the system's response is obtained simply by adding the contact-impact forces to the multi-body system's equations of motion as external generalized forces. This approach provides accurate results, insofar as the equations of motion are integrated over the period of contact. It, thus, accounts for the changes in the configuration and velocities of the system during that contact. In the proposed methodology, the clearance is of crucial importance in the joint kinematics, due to the role that the system's configuration geometric conditions play to define if the joint elements are or not in contact.

Mechanical systems with rigid and flexible bodies and with non-ideal joints have been treated in the past. Studies have considered joint compliance and friction, but without clearances [8]. Methods for modelling joint connections and external impacts using restitution coefficient and momentum have also been proposed in the past $[\mathbf{9}, \mathbf{1 0}]$. While such methods offer the advantage of relatively low computational effort over techniques that explicitly model the joint impact not only they do not provide the time history of the values of these forces, which are important from the design point of view, but also they assume that no change in the realistic orientation between contacting bodies occur during impact, which is not true for clearance joints. The measurement of the influence that joint clearances have on the kinematic and dynamic performance of a mechanism provides a useful criterion for judging the suitability of the mechanism to perform a given task. 
Important aspects in the modelling of spatial mechanisms such as the link's flexibility or the joints' lubrication are not discussed here as they fall out of the scope of this work. A detailed discussion on the modelling aspects of flexible multi-body systems can be found on the work by Ambrósio [11], whereas the modelling of clearance joints with tribological aspects is discussed in the work by Flores et al. [5, 6].

The main purpose of this work is to develop and demonstrate an effective methodology for spatial multi-body systems, including revolute joints with clearance. Owing to their relevance for this work, some aspects of the multi-body formulation for spatial systems, based on the Cartesian coordinates, are reviewed here to introduce the basic aspects on the dynamic modelling of spatial multi-body systems with joint clearances $[12,13]$. A brief description of the ideal or perfect spatial revolute joint is presented. The numerical effectiveness of the method is shown in the application to a double pendulum in which a spatial revolute with clearance exists.

\section{MODELLING SPATIAL REVOLUTE JOINTS WITH CLEARANCE}

The use of spatial revolute joint clearances in the multi-body system formulation requires that a suitable mathematical description is developed. Figure 3 shows a representation of a spatial revolute joint with clearance that connects bodies $i$ and $j$. The bearing is part of body $i$ and the journal is part of body $j$. The difference in radius between the bearing and journal, $c=R_{i}-R_{j}$, defines the radial clearance, in which $R_{\mathrm{i}}$ and $R_{\mathrm{j}}$ are the bearing and journal radii, respectively. The centre of mass of bodies $i$ and $j$ are $O_{\mathrm{i}}$ and $O_{\mathrm{j}}$, respectively. A body-fixed coordinate system $\xi \eta \zeta$ is attached at the centre of mass of each body, whereas the $X Y Z$ denotes the global coordinate system. The geometric centre of the bearing is located at point $P$ which, together with point $Q$, defines the joint/bearing axis, whereas points $V$ and $W$ on body $j$ define the journal axis. These points are located at the top and bottom of journal bases, so that the distance between $V$ and $W$ defines the length of the joint.

Figure 4 shows two different scenarios for the contact between the journal and the bearing. For simplicity, in Fig. 4, only the journal and bearing are represented. In the present methodology, only the top and the bottom journal bases are considered for contact detection between the journal and bearing elements. Furthermore, it is assumed that the clearance is much smaller than the dimensions of the bodies, so that the angle between the bearing and journal axes, represented by $\theta$ in Fig. 4(a), is

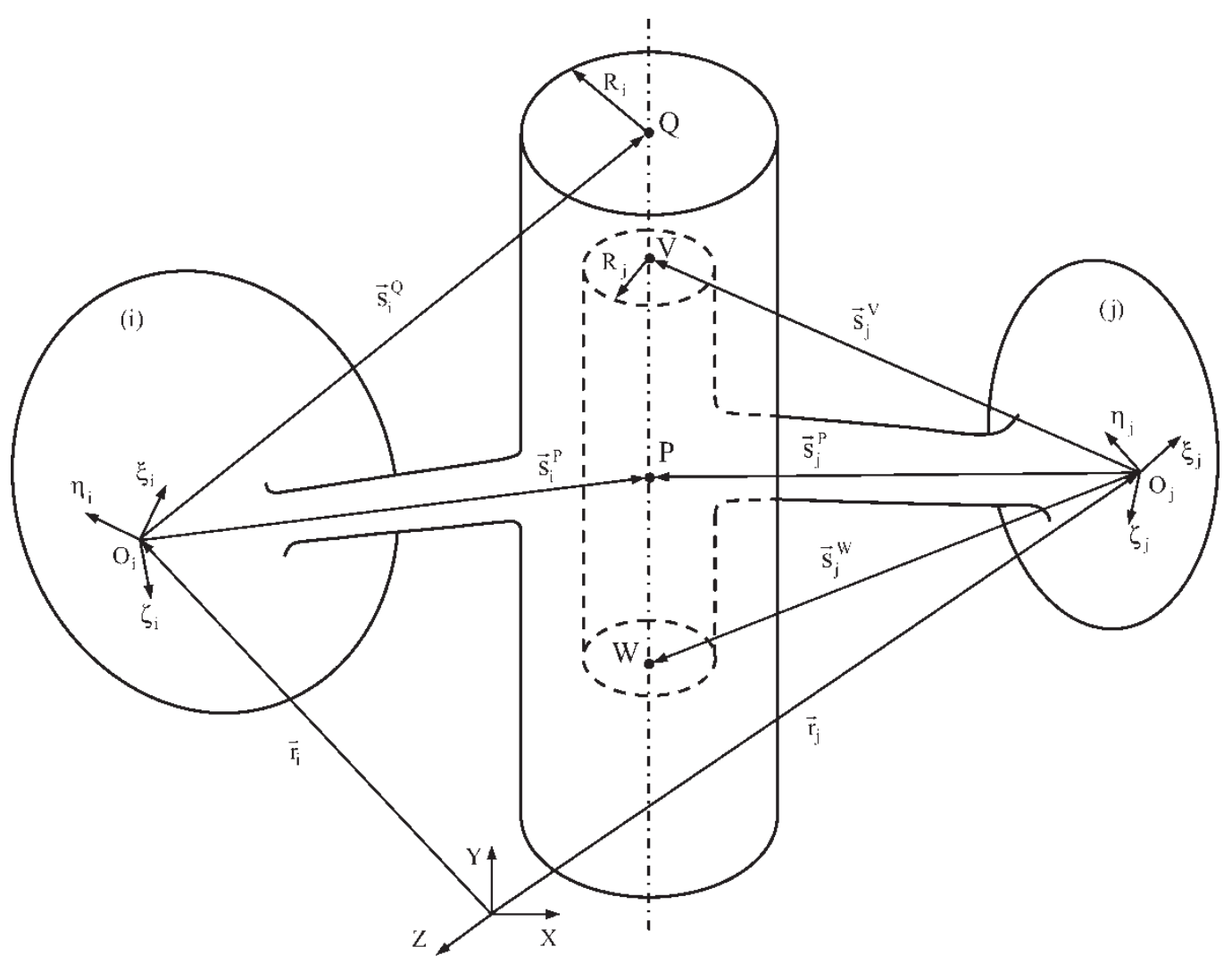

Fig. 3 General configuration of a spatial revolute joint with clearance in a multi-body system 


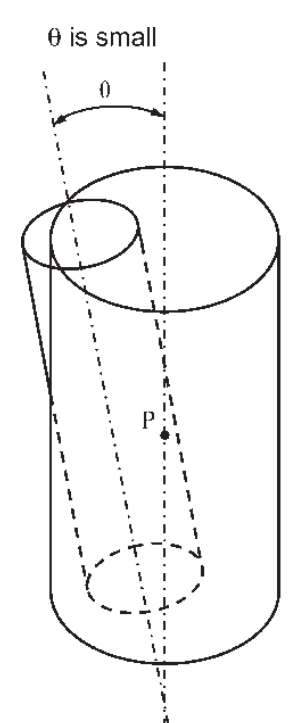

(a)

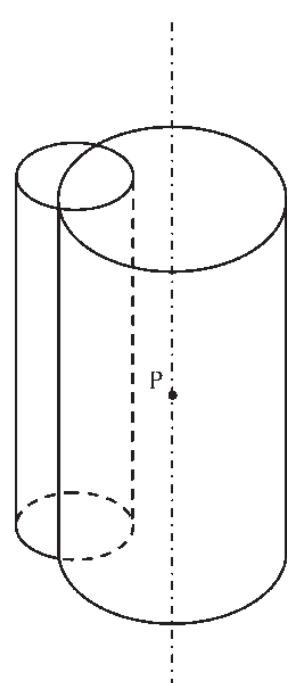

(b)

Fig. 4 Two different scenarios for contact between the journal and bearing wall: (a) only one journal base (top) contacts with the bearing wall, and (b) both bases (top and bottom) contact with the bearing wall

very small and, consequently, both top and bottom journal bases can be assumed to be parallel between them and perpendicular to the joint axis.

Assuming a local coordinate system $(\xi \eta \zeta)_{\mathrm{r}}$ associated with the revolute joint axis located at point $P$,

the unit coordinate vectors defined along the local axes are $\boldsymbol{u}_{\xi_{\mathrm{r}}}^{\prime}, \boldsymbol{u}_{\eta_{\mathrm{r}}}^{\prime}$, and $\boldsymbol{u}_{\zeta_{\mathrm{r}}}^{\prime}$, as illustrated in Fig. 5. The unit vector along the $\zeta_{\mathrm{r}}$-axis, $\boldsymbol{u}_{\zeta_{\mathrm{r}}}^{\prime}$, is evaluated as

$$
\boldsymbol{u}_{\zeta_{\mathrm{r}}}^{\prime}=\frac{\boldsymbol{s}_{i}^{\mathbf{Q}^{\mathrm{Q}}}-\boldsymbol{s}_{i}^{\prime \mathrm{P}}}{\left\|\boldsymbol{s}_{i}^{\mathrm{Q}}-\boldsymbol{s}_{i}^{\prime \mathrm{P}}\right\|}
$$

where both vectors $\boldsymbol{s}_{i}^{\prime \mathrm{P}}$ and $\boldsymbol{s}_{i}^{\mathrm{Q}}$ are described in the local coordinate system of body $i$. The remaining two unit vectors are evaluated according to

$$
\left\{\begin{array}{l}
\boldsymbol{u}_{\xi_{\mathrm{r}}}^{\prime}=\boldsymbol{u}_{\xi_{\mathrm{i}}}^{\prime} \\
\boldsymbol{u}_{\eta_{\mathrm{r}}}^{\prime}=\boldsymbol{u}_{\eta_{\mathrm{i}}}^{\prime}
\end{array} \text { if } \boldsymbol{u}_{\zeta_{\mathrm{r}}}^{\prime}=\boldsymbol{u}_{\zeta_{\mathrm{i}}}^{\prime}\right.
$$

or

$$
\left\{\begin{array}{l}
\boldsymbol{u}_{\xi_{\mathrm{r}}}^{\prime}=\tilde{\boldsymbol{u}}_{\zeta_{\mathrm{r}}}^{\prime} \boldsymbol{u}_{\zeta_{\mathrm{i}}}^{\prime} \\
\boldsymbol{u}_{\eta_{\mathrm{r}}}^{\prime}=\tilde{\boldsymbol{u}}_{\zeta_{\mathrm{r}}}^{\prime} \boldsymbol{u}_{\xi_{\mathrm{r}}}^{\prime}
\end{array} \text { if } \boldsymbol{u}_{\zeta_{\mathrm{r}}}^{\prime} \neq \boldsymbol{u}_{\zeta_{\mathrm{i}}}^{\prime}\right.
$$

The transformation matrix $\mathbf{A}_{\mathrm{ri}}$ that transforms the local components $(\xi \eta \zeta)_{\mathrm{r}}$ into the local coordinate system $(\xi \eta \zeta)_{\mathrm{i}}$ is expressed as $[\mathbf{1 2}]$

$$
\mathbf{A}_{\mathrm{r} i}=\left[\begin{array}{lll}
\boldsymbol{u}_{\xi_{\mathrm{r}}}^{\prime} & \boldsymbol{u}_{\eta_{\mathrm{r}}}^{\prime} & \boldsymbol{u}_{\zeta_{\mathrm{r}}}^{\prime}
\end{array}\right]^{\mathrm{T}}
$$

Note that the transformation matrix defined by equation (4) is constant. Furthermore, if $\boldsymbol{u}_{\zeta_{r}}^{\prime}=\boldsymbol{u}_{\zeta_{\mathrm{i}}}^{\prime}$ the transformation matrix $\mathbf{A}_{\mathrm{r} i}$ is equal to the identity

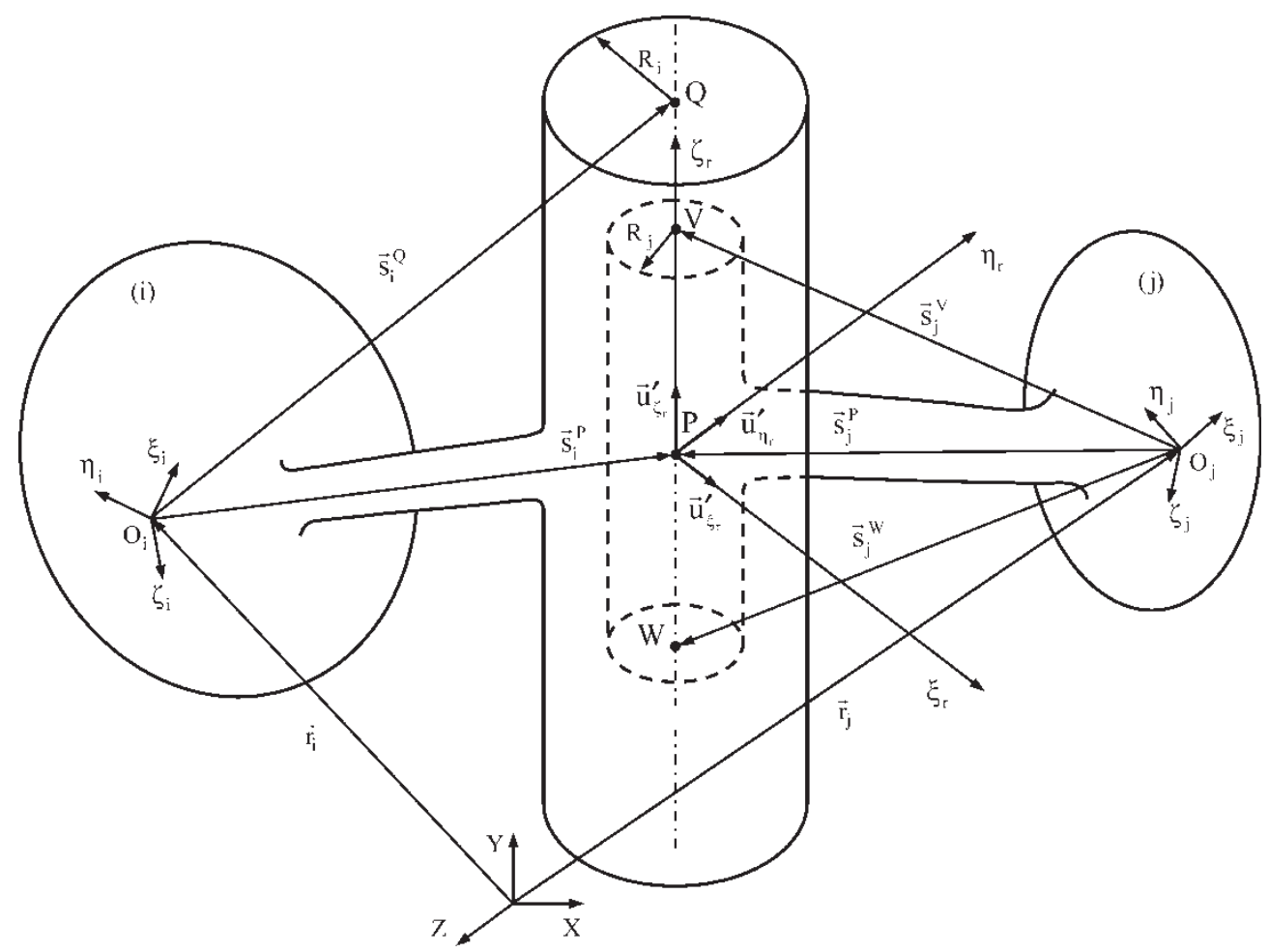

Fig. 5 Local coordinate system associated with the revolute joint clearance and the respective unit vectors 
matrix I. Thus, the matrix that transforms the local vectors $(\xi \eta \zeta)_{\mathrm{r}}$ into the global reference system $X Y Z$ is given by

$$
\mathbf{A}_{\mathrm{r}}=\mathbf{A}_{i} \mathbf{A}_{\mathrm{r} i}
$$

The global position of the origin of the local coordinate system $(\xi \eta \zeta)_{\mathrm{r}}$ is

$$
\boldsymbol{r}_{i}^{\mathrm{P}}=\boldsymbol{r}_{i}+\mathbf{A}_{i} \boldsymbol{s}_{i}^{\prime \mathrm{P}}
$$

In order to define the relative position between the journal and bearing, it is first necessary to express the vectors $\boldsymbol{s}_{j}^{\mathrm{V}}$ and $\boldsymbol{s}_{j}^{\mathrm{W}}$ in the local coordinate system associated with the joint $(\xi \eta \zeta)_{\text {r }}$. From Fig. 5, the global coordinates of points $\mathrm{V}$ and $\mathrm{W}$ with respect to the inertial reference frame are expressed as

$$
\begin{aligned}
& \boldsymbol{r}_{j}^{\mathrm{V}}=\boldsymbol{r}_{j}+\mathbf{A}_{j} \boldsymbol{s}_{j}^{\prime} \mathrm{V} \\
& \boldsymbol{r}_{j}^{\mathrm{W}}=\boldsymbol{r}_{j}+\mathbf{A}_{j} \boldsymbol{s}_{j}^{\prime} \mathrm{W}
\end{aligned}
$$

Thus, vectors $\boldsymbol{s}_{j}^{\mathrm{V}}$ and $\boldsymbol{s}_{j}^{\mathrm{W}}$ expressed in the global coordinate system are

$$
\begin{aligned}
& \boldsymbol{s}_{\mathrm{r}}^{\mathrm{V}}=\boldsymbol{r}_{j}^{\mathrm{V}}-\boldsymbol{r}_{i}^{\mathrm{P}} \\
& \boldsymbol{s}_{\mathrm{r}}^{\mathrm{W}}=\boldsymbol{r}_{j}^{\mathrm{W}}-\boldsymbol{r}_{i}^{\mathrm{P}}
\end{aligned}
$$

When expressed in the local coordinate system of the joint, these vectors are given by

$$
\begin{aligned}
& \boldsymbol{s}_{\mathrm{r}}^{\prime \mathrm{V}}=\mathbf{A}_{\mathrm{r}}^{\mathrm{T}} \boldsymbol{s}_{\mathrm{r}}^{\mathrm{V}} \\
& \boldsymbol{s}_{\mathrm{r}}^{\prime \mathrm{W}}=\mathbf{A}_{\mathrm{r}}^{\mathrm{T}} \boldsymbol{s}_{\mathrm{r}}^{\mathrm{W}}
\end{aligned}
$$

The vectors given by equations (9) define the coordinates of points $\mathrm{V}$ and $\mathrm{W}$ of the journal, expressed in terms of the local coordinate system associated with the joint, that is $(\xi \eta \zeta)_{\mathrm{r}}$.

Figure 6 depicts a system's configuration in which both top and bottom journal bases contact with the bearing wall. The eccentricity vectors at the top and bottom journal bases, $\boldsymbol{e}_{\mathrm{r}}^{\mathrm{V}}$ and $\boldsymbol{e}_{\mathrm{r}}^{\mathrm{W}}$, are given by the projection of the vectors $\boldsymbol{s}_{\mathrm{r}}^{\mathrm{V}}$ and $\boldsymbol{s}_{\mathrm{r}}^{\mathrm{W}}$ onto the local axes $\xi_{\mathrm{r}}$ and $\eta_{\mathrm{r}}$

$$
\begin{array}{lll}
\boldsymbol{e}_{\mathrm{r}}^{\mathrm{V}}=\left\{\left(\boldsymbol{s}_{\mathrm{r}}^{\prime}\right)_{\xi_{\mathrm{r}}}^{\mathrm{V}}\right. & \left(\boldsymbol{s}_{\mathrm{r}}^{\prime \mathrm{V}}\right)_{\eta_{\mathrm{r}}} & 0\}^{\mathrm{T}} \\
\boldsymbol{e}_{\mathrm{r}}^{\mathrm{W}}=\left\{\begin{array}{lll}
\left(\boldsymbol{s}_{\mathrm{r}}^{\prime \mathrm{W}}\right)_{\xi_{\mathrm{r}}} & \left(\boldsymbol{s}_{\mathrm{r}}^{\prime \mathrm{W}}\right)_{\eta_{\mathrm{r}}} & 0
\end{array}\right\}^{\mathrm{T}}
\end{array}
$$

The magnitudes of the eccentricity vectors are
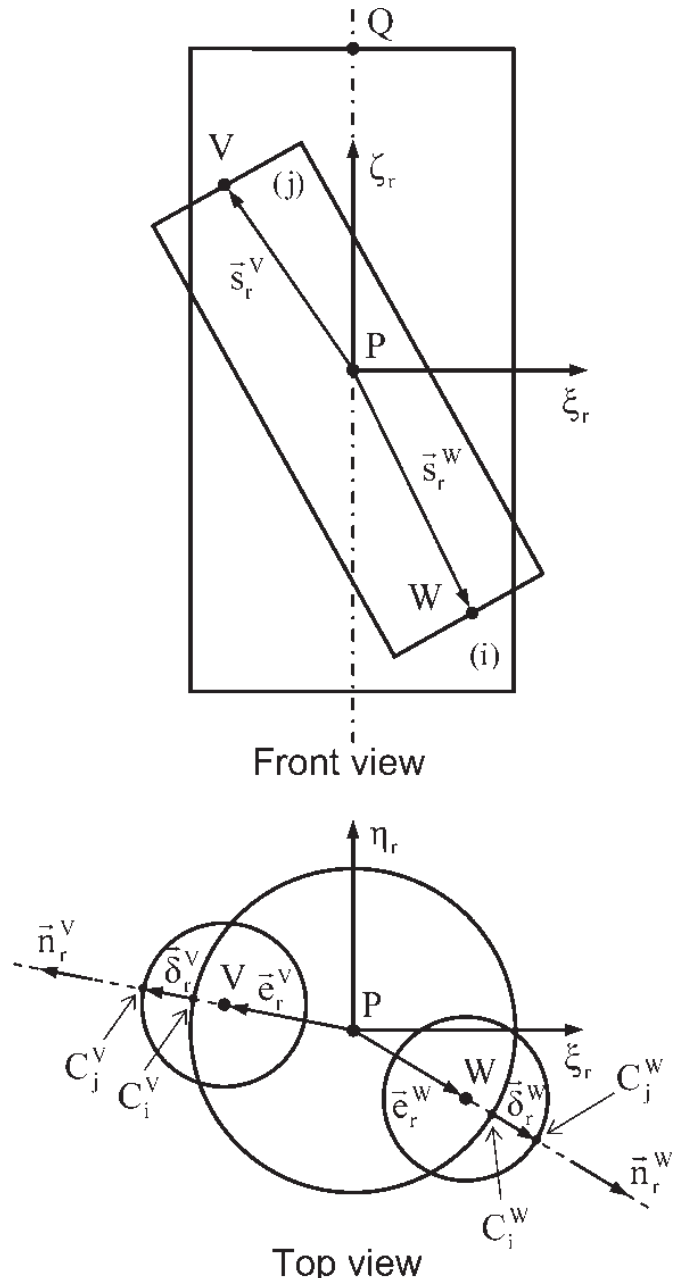

Fig. 6 Front and top views of the top and bottom journal bases at the point of contact with the bearing wall

evaluated as

$$
\begin{aligned}
\boldsymbol{e}_{\mathrm{r}}^{\mathrm{V}} & =\sqrt{\left(\boldsymbol{e}_{\mathrm{r}}^{\mathrm{V}}\right)^{\mathrm{T}} \boldsymbol{e}_{\mathrm{r}}^{\mathrm{V}}} \\
\boldsymbol{e}_{\mathrm{r}}^{\mathrm{W}} & =\sqrt{\left(\boldsymbol{e}_{\mathrm{r}}^{\mathrm{W}}\right)^{\mathrm{T}} \boldsymbol{e}_{\mathrm{r}}^{\mathrm{W}}}
\end{aligned}
$$

The unit vectors, $\boldsymbol{n}_{\mathrm{r}}^{\mathrm{V}}$ and $\boldsymbol{n}_{\mathrm{r}}^{\mathrm{W}}$, normal to the planes of contact where the top and bottom journal bases touch the bearing wall, are evaluated as (Fig. 6)

$$
\begin{aligned}
& \boldsymbol{n}_{\mathrm{r}}^{\mathrm{V}}=\frac{\boldsymbol{e}_{\mathrm{r}}^{\mathrm{V}}}{\left\|\boldsymbol{e}_{\mathrm{r}}^{\mathrm{V}}\right\|} \\
& \boldsymbol{n}_{\mathrm{r}}^{\mathrm{W}}=\frac{\boldsymbol{e}_{\mathrm{r}}^{\mathrm{W}}}{\left\|\boldsymbol{e}_{\mathrm{r}}^{\mathrm{W}}\right\|}
\end{aligned}
$$

Referring to Fig. 6, the penetrations due to the contact between the journal bases and bearing wall 
are calculated as

$$
\begin{aligned}
& \delta_{\mathrm{r}}^{\mathrm{V}}=\boldsymbol{e}_{\mathrm{r}}^{\mathrm{V}}-c \\
& \delta_{\mathrm{r}}^{\mathrm{W}}=\boldsymbol{e}_{\mathrm{r}}^{\mathrm{W}}-c
\end{aligned}
$$

where $\boldsymbol{e}_{\mathrm{r}}^{\mathrm{V}}$ and $\boldsymbol{e}_{\mathrm{r}}^{\mathrm{W}}$ are, respectively, the modules of the eccentricity vectors at the top and bottom journal bases, and $c$ is the radial clearance.

Considering $C_{i}^{\mathrm{V}}, C_{j}^{\mathrm{V}}, C_{i}^{\mathrm{W}}$, and $C_{j}^{\mathrm{W}}$ to be the potential contact points on bodies $i$ and $j$, the global position of such points is evaluated as

$$
\begin{aligned}
& \boldsymbol{r}_{k}^{C_{k}^{\mathrm{V}}}=\boldsymbol{r}_{k}^{\mathrm{P}}+\mathbf{A}_{k} \boldsymbol{s}_{k}^{C_{k}^{\mathrm{V}}} \\
& \boldsymbol{r}_{k}^{C_{k}^{\mathrm{W}}}=\boldsymbol{r}_{k}^{\mathrm{P}}+\mathbf{A}_{k} \boldsymbol{s}_{k}^{\prime} C_{k}^{\mathrm{W}}
\end{aligned} \quad(k=i, j)
$$

where vectors $\boldsymbol{s}_{k}^{C_{k}^{\mathrm{V}}}$ and $\boldsymbol{s}_{k}^{C_{k}^{\mathrm{W}}}(k=i, j)$, are the local coordinates of vectors $\boldsymbol{s}_{k}^{C_{k}^{V}}$ and $\boldsymbol{s}_{k}^{C_{k}^{W}}$ on bodies $i$ and $j$, expressed in terms of the local coordinate system of each body. These vectors are only defined in the local coordinate system of the joint $(\xi \eta \zeta)_{\mathrm{r}}$, and are expressed as

$$
\begin{aligned}
& \boldsymbol{s}_{\mathrm{r}}^{C_{i}^{\mathrm{V}}}=\left\{\begin{array}{lll}
0 & 0 & \left(\boldsymbol{s}_{\mathrm{r}}^{\prime \mathrm{V}}\right)_{\zeta_{\mathrm{r}}}
\end{array}\right\}^{\mathrm{T}}+R_{i} \boldsymbol{n}_{\mathrm{r}}^{\mathrm{V}} \\
& \boldsymbol{s}_{\mathrm{r}}^{C_{j}^{\mathrm{V}}}=\boldsymbol{s}_{\mathrm{r}}^{\mathrm{V}}+R_{j} \boldsymbol{n}_{\mathrm{r}}^{\mathrm{V}} \\
& \boldsymbol{s}_{\mathrm{r}}^{C_{i}^{\mathrm{W}}}=\left\{\begin{array}{lll}
0 & 0 & \left(\boldsymbol{s}_{\mathrm{r}}^{\prime \mathrm{W}}\right)_{\zeta_{\mathrm{r}}}
\end{array}\right\}^{\mathrm{T}}+R_{i} \boldsymbol{n}_{r}^{\mathrm{W}} \\
& \boldsymbol{s}_{r}^{\prime} \mathrm{C}_{j}^{\mathrm{W}}=\boldsymbol{s}_{r}^{\prime \mathrm{W}}+R_{j} \boldsymbol{n}_{r}^{\mathrm{W}}
\end{aligned}
$$

These vectors are written in terms of the local coordinate systems associated with bodies $i$ and $j$ as

$$
\begin{aligned}
& \boldsymbol{s}_{i}^{\prime C_{i}^{\mathrm{V}}}=\mathbf{A}_{\mathrm{ri}} \boldsymbol{s}_{\mathrm{r}}^{\prime_{i}^{\mathrm{V}}} \\
& \boldsymbol{s}_{j}^{C_{j}^{\mathrm{V}}}=\mathbf{A}_{j}^{\mathrm{T}} \boldsymbol{A}_{\mathrm{r}} \boldsymbol{s}_{\mathrm{r}}^{C_{j}^{\mathrm{V}}} \\
& \boldsymbol{s}_{i}^{\prime C_{i}^{\mathrm{W}}}=\mathbf{A}_{\mathrm{ri}} \boldsymbol{s}_{\mathrm{r}}^{\prime C_{i}^{\mathrm{W}}} \\
& \boldsymbol{s}_{j}^{C_{j}^{\mathrm{W}}}=\mathbf{A}_{j}^{\mathrm{T}} \boldsymbol{A}_{\mathrm{r}} \boldsymbol{s}_{\mathrm{r}}^{\prime_{i}} C_{j}^{\mathrm{W}}
\end{aligned}
$$

The contact force model used in this work, to represent the interactions between the journal and bearing, requires that the impact velocity is known. These impact velocities are obtained by differentiating equations (14) with respect to time, yielding

$$
\begin{aligned}
& \dot{\boldsymbol{r}}_{k}^{C_{k}^{\mathrm{V}}}=\dot{\boldsymbol{r}}_{k}^{\mathrm{P}}+\mathbf{A}_{k} \tilde{\boldsymbol{\omega}}_{k}^{\prime} \boldsymbol{s}_{k}^{\prime} C_{k}^{\mathrm{V}} \\
& \dot{\boldsymbol{r}}_{k}^{C_{k}^{\mathrm{W}}}=\dot{\boldsymbol{r}}_{k}^{\mathrm{P}}+\mathbf{A}_{k} \tilde{\boldsymbol{\omega}}_{k}^{\prime} \boldsymbol{s}_{k}^{\prime} C_{k}^{\mathrm{W}}
\end{aligned} \quad(k=i, j)
$$

The relative impact velocities at the contact points are

$$
\begin{aligned}
& \Delta \dot{\boldsymbol{r}}^{\mathrm{V}}=\dot{\boldsymbol{r}}_{j}^{C_{j}^{\mathrm{V}}}-\dot{\boldsymbol{r}}_{i}^{C_{i}^{\mathrm{V}}} \\
& \Delta \dot{\boldsymbol{r}}^{\mathrm{W}}=\dot{\boldsymbol{r}}_{j}^{C_{j}^{\mathrm{W}}}-\dot{\boldsymbol{r}}_{i}^{C_{i}^{\mathrm{W}}}
\end{aligned}
$$

The relative velocities of the contact points are projected onto the direction of the penetration, yielding to the relative normal velocities, $\dot{\delta}_{\mathrm{r}}^{\mathrm{V}}$ and $\dot{\delta}_{\mathrm{r}}^{\mathrm{W}}$, as shown in Fig. 7. These velocities are evaluated by

$$
\begin{aligned}
& \dot{\delta}_{\mathrm{r}}^{\mathrm{V}}=\left(\Delta \dot{\boldsymbol{r}}^{\mathrm{V}}\right)^{\mathrm{T}} \boldsymbol{n}_{r}^{\mathrm{V}} \\
& \dot{\delta}_{\mathrm{r}}^{\mathrm{W}}=\left(\Delta \dot{\boldsymbol{r}}^{\mathrm{W}}\right)^{\mathrm{T}} \boldsymbol{n}_{\mathrm{r}}^{\mathrm{W}}
\end{aligned}
$$

When contact between the journal and bearing takes place, impact forces act at the contact points. The normal contact forces are denoted by $\boldsymbol{f}_{\mathrm{N}}$, while, assuming that the contact is not frictionless, the tangential forces are $\boldsymbol{f}_{\mathrm{T}}$. Since these forces do not act through the centre of mass of the bodies $i$ and $j$, the moment components for each body need to be evaluated. For convenience and simplicity, the bodies are presented separately in Fig. 8 and only the force components that act at the top journal base are illustrated. According to Fig. 8, the forces and moments working on the centre of mass of body $i$ are given by

$$
\begin{aligned}
& \boldsymbol{f}_{i}=\boldsymbol{f}_{\mathrm{N}}+\boldsymbol{f}_{\mathrm{T}} \\
& \mathbf{m}_{i}^{\prime}=\mathbf{A}_{i}^{\mathrm{T}}\left(\tilde{\boldsymbol{s}}_{i}^{\mathrm{C}^{\mathrm{V}}} \boldsymbol{f}_{i}\right)
\end{aligned}
$$

The forces and moments corresponding to the body $j$ are written as

$$
\begin{aligned}
& \boldsymbol{f}_{j}=-\boldsymbol{f}_{i} \\
& \mathbf{m}_{j}^{\prime}=\mathbf{A}_{j}^{\mathrm{T}}\left(\tilde{\boldsymbol{s}}_{j}^{\mathrm{V}} \boldsymbol{f}_{j}\right)
\end{aligned}
$$

Note that the moments acting on the rigid bodies are expressed in body fixed coordinates. This is due to the multi-body formulation used in this work, which follows closely that proposed by Nikravesh [12].

\section{COMPUTATIONAL IMPLEMENTATION}

Since the formulation of the spatial revolute joint involves a good deal of mathematical manipulation, it is convenient to summarize the main steps in an appropriate algorithm. This algorithm, presented in 


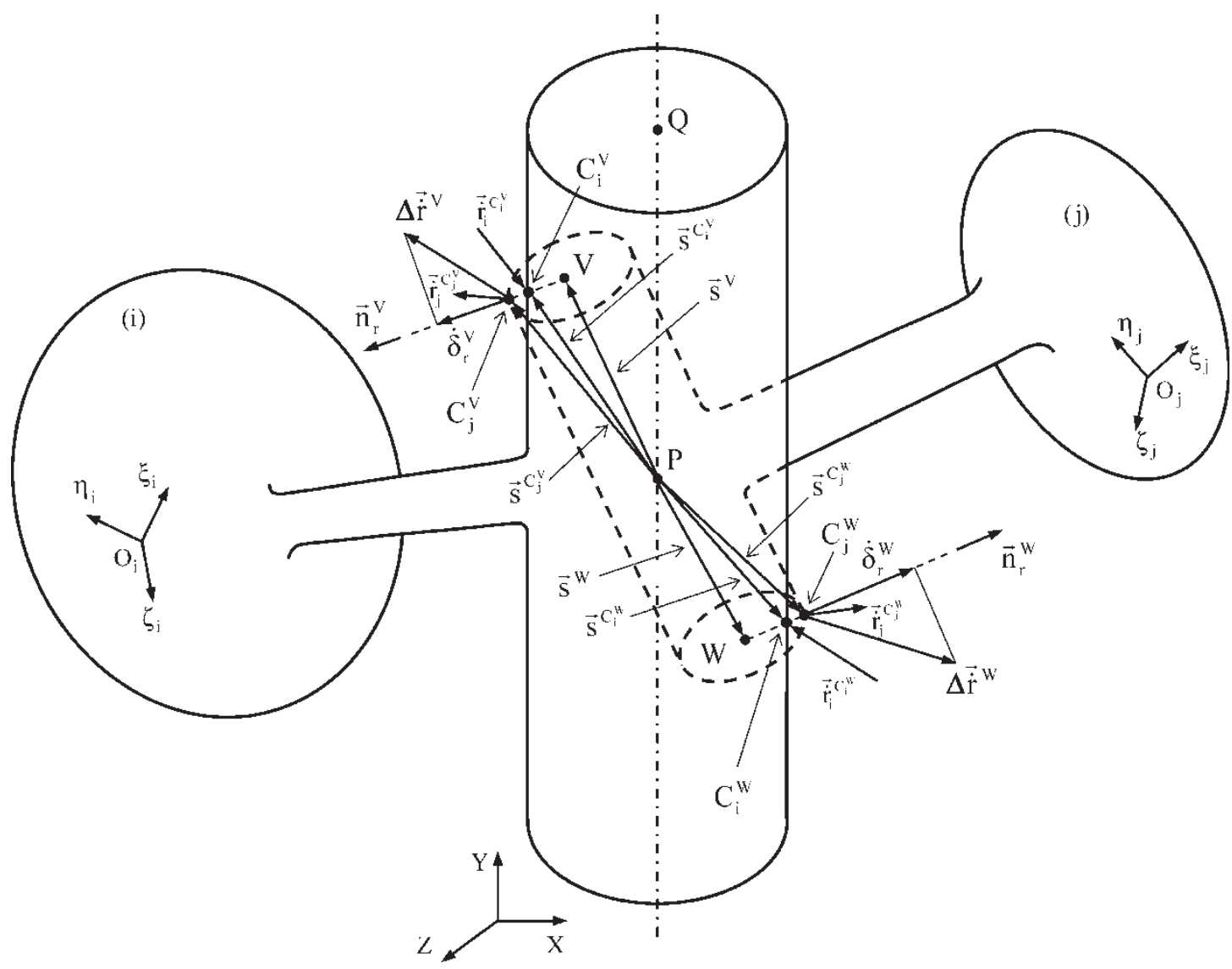

Fig. 7 Location of the contact points and representation of impact velocities

the flowchart of Fig. 9, is developed in the framework of the multi-body methodology and can be synthesized condensed in the following steps.

1. Start at instant of time $t^{0}$, with given initial conditions for positions $\boldsymbol{q}^{0}$ and velocities $\dot{\boldsymbol{q}}^{0}$.
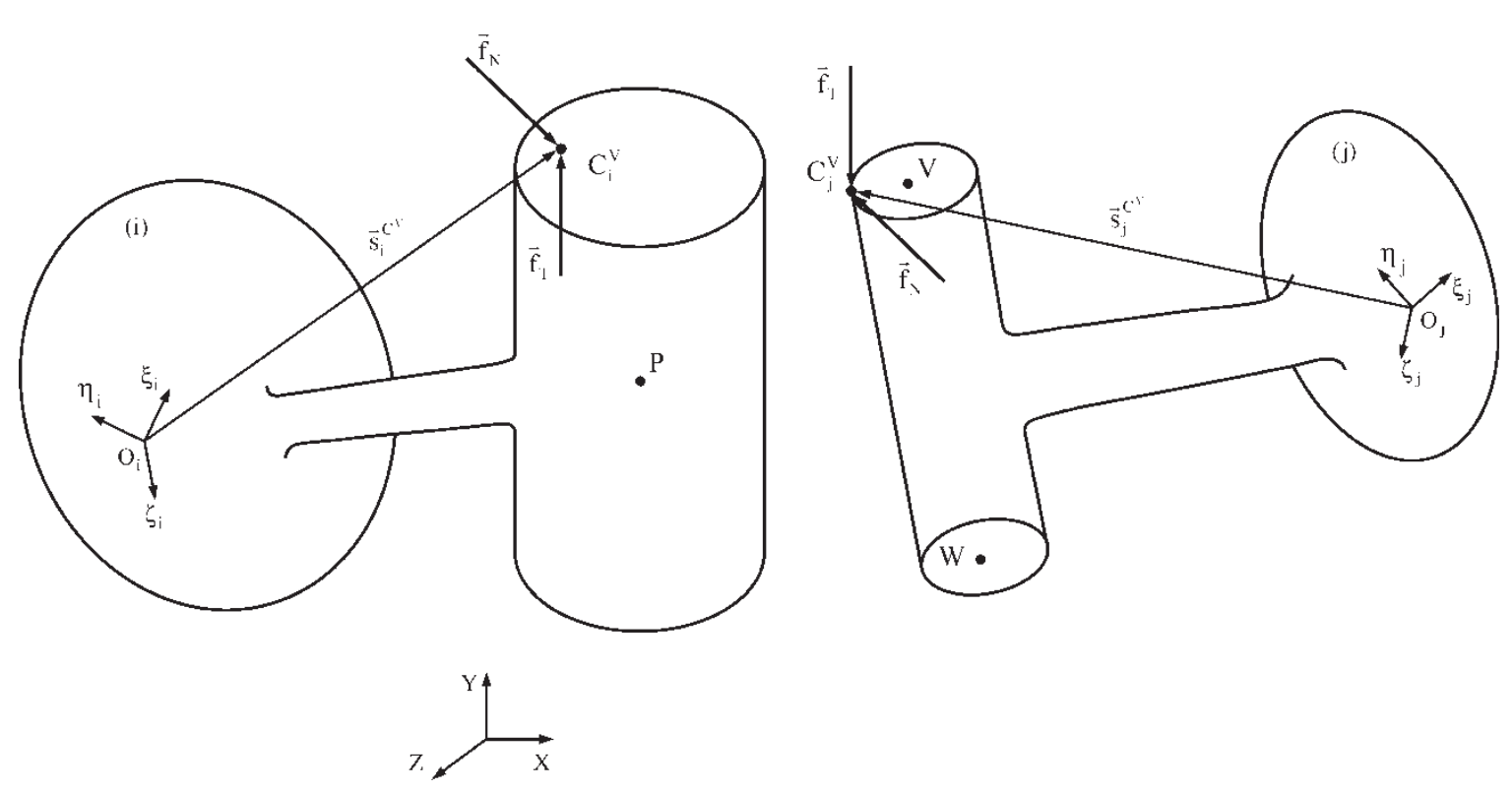

2. Define the location of point $P_{i}, P_{j}, Q_{i}, V_{j}$, and $W_{j}$, necessary to describe the revolute joint clearance. Define joint and material properties $\left(R_{\mathrm{B}}\right.$, $E_{\mathrm{B}}, \nu_{\mathrm{B}}, R_{\mathrm{J}}, E_{\mathrm{J}}$, and $\left.\nu_{\mathrm{J}}\right)$.

3. Compute the local coordinate system associated with the joint, $(\xi \eta \zeta)_{\mathrm{r}}$ and evaluate the unit

Fig. 8 Contact forces defined at the points of contact between the journal top base and bearing wall 


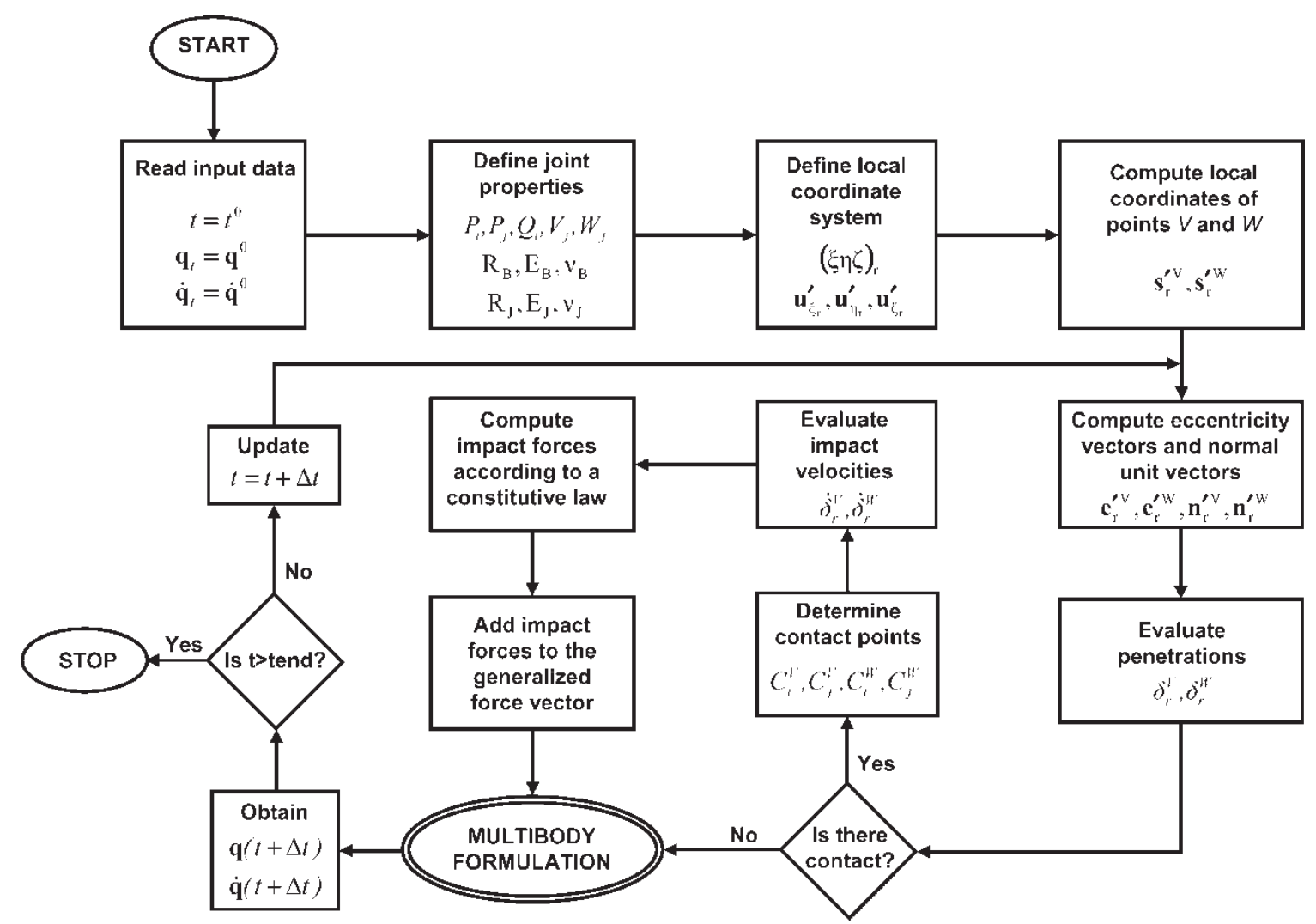

Fig. 9 Algorithm proposed to model spatial revolute joint with clearance in multi-body systems

coordinates vectors along each axis, that is $\boldsymbol{u}_{\xi_{r}}^{\prime}$, $\boldsymbol{u}_{\eta_{\mathrm{r}}}^{\prime}$, and $\boldsymbol{u}_{\zeta_{\mathrm{r}}}^{\prime}$ using equations (1) to (3).

4. Evaluate the local coordinates of the geometrical centres of the top and bottom bases in the $(\xi \eta \zeta)_{\mathrm{r}}$ coordinate system using equations (9).

5. Compute the eccentricity vectors $\boldsymbol{e}_{\mathrm{r}}^{\mathrm{V}}$ and $\boldsymbol{e}_{\mathrm{r}}^{\mathrm{W}}$ as well as the unit vectors that define the direction of impact $\boldsymbol{n}_{\mathrm{r}}^{\mathrm{V}}$ and $\boldsymbol{n}_{\mathrm{r}}^{\mathrm{W}}$ through equations (10) to (12).

6. Evaluate the penetrations $\delta_{\mathrm{r}}^{\mathrm{V}}$ and $\delta_{\mathrm{r}}^{\mathrm{W}}$ with equations (13).

7. Check for contact: if there is contact, determine the contact points using equations (14), evaluate the impact velocities with equations (21), compute the impact forces by Equations (22) to (23), and add the impact forces to the equations of motion.

8. Apply a multi-body formulation in order to obtain the new generalized positions and velocities of the system for time step $t+\Delta t$.

9. Update the system time variable.

10. Go to step 5 and proceed with the whole process for the new time step, until the final time for the analysis is reached.

\section{CONTACT FORCE MODEL}

In dealing with revolute joint clearances, it is essential to define how the journal and bearing surfaces contact each other and, consequently, what is the most adequate contact force model. The contact force model is expected to contribute, if possible, to the stable integration of the equations of motion of the multi-body system.

The elastic force developed in the contact collision between the journal and bearing surfaces can be modelled by applying the Hertz contact law, which is based on the elasticity theory [14]. The Hertz contact model represents the contact force as a nonlinear function of the penetration [15]. Hunt and Crossley [16] proposed a non-linear viscous-elastic model to represent the damping force, which simulates the energy transferred during the impact process. Based on the work of Hunt and Crossley, Lankarani and Nikravesh [7] presented a continuous contact force model in which a hysteretic damping factor is incorporated in order to account for the energy dissipation. This contact force model is expressed as

$$
F_{\mathrm{N}}=K \delta^{n}\left[1+\frac{3\left(1-c_{\mathrm{e}}^{2}\right)}{4} \frac{\dot{\delta}}{\dot{\delta}^{(-)}}\right]
$$

where $K$ is the generalized stiffness parameter, $n$ an exponent equal to $1.5, c_{\mathrm{e}}$ the restitution coefficient, $\dot{\delta}$ the relative penetration velocity, and $\dot{\delta}^{(-)}$the initial impact velocity. Equation (24) is used to simulate 
the impact because it accounts for energy dissipation and exhibits good numerical stability at low impact velocities. The proportionality constant $K$ is calculated for the case of internal contact between two cylinders according to the model proposed by Ravn [4].

\section{FORMULATION OF SPATIAL MULTI-BODY SYSTEMS}

The formulation of the equations of motion to the spatial dynamic analysis of multi-body systems is reviewed to provide a framework to the application of the method proposed. A simple and brief description of the standard mechanical joints of spatial multi-body systems is presented, namely of the ideal spherical and revolute joints, to emphasize the differences with respect to joints with clearance. These two types of joints fall under the category of holonomic constraints as they can be explicitly expressed in terms of the positions of the rigid bodies [12]. A kinematic joint imposes certain conditions on the relative motion between the adjacent bodies that it comprises. When these conditions are expressed in analytical form, they are called constraint equations. In a simple way, a constraint is any condition that reduces the number of degrees of freedom in a system.

A ideal spherical joint, also known as by ball and socket joint, illustrated in Fig. 10, constrains the relative translations between two adjacent bodies $i$ and $j$, allowing only three relative rotations. Thus, the centre of the spherical joint, point $P$, has constant coordinates with respect to any of the local coordinates systems of the connected bodies, i.e. a spherical joint is defined by the condition that the point $P_{i}$ on body $i$ coincides with the point $P_{j}$ on body $j$. This condition is simply the spherical constraint, which can be written in a scalar form as [12]

$$
\boldsymbol{\Phi}^{(s, 3)} \equiv \boldsymbol{r}_{i}+\mathbf{A}_{i} \boldsymbol{s}_{i}^{\mathrm{P}}-\boldsymbol{r}_{j}-\mathbf{A}_{j} \boldsymbol{s}_{j}^{\prime \mathrm{P}}=0
$$

The three scalar constraint equations implied by equation (25) restrict only the relative position of points $P_{i}$ and $P_{j}$. There are, therefore, three relative degrees of freedom between two bodies that are connected by a perfect spherical joint.

An ideal three-dimensional revolute or rotational joint between bodies $i$ and $j$, shown in Fig. 11, is built with a journal-bearing that allows a relative rotation about a common axis, but precludes relative translation along this axis. Any point located on the revolute joint axis has constant coordinates, when expressed in the local coordinate systems of the connected bodies. Thus, equation (25) can be imposed on an arbitrary point $P$ on the joint axis. Two other

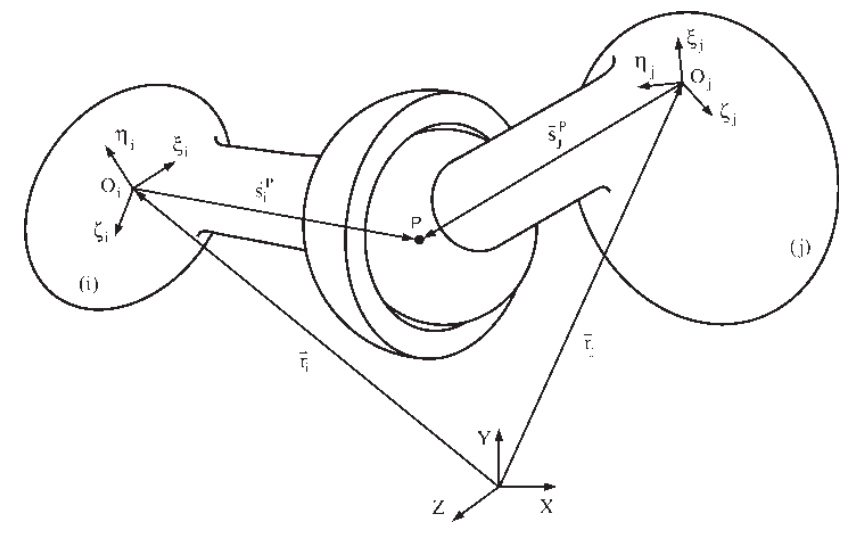

Fig. 10 Perfect spherical joint in a multi-body system

points $Q_{i}$ on body $i$ and $Q_{j}$ on body $j$ are also arbitrarily chosen on the joint axis. It is clear that vectors $\boldsymbol{s}_{i}$ and $\boldsymbol{s}_{j}$ must remain parallel. Therefore, there are five constraint equations for a spatial revolute joint [11]

$$
\boldsymbol{\Phi}^{(r, 5)} \equiv\left\{\begin{array}{c}
\boldsymbol{r}_{i}+\mathbf{A}_{i} \boldsymbol{s}_{i}^{\prime}{ }_{i}-\boldsymbol{r}_{j}-\mathbf{A}_{j} \boldsymbol{s}_{j}^{\prime} \mathrm{P}=0 \\
\tilde{\boldsymbol{s}}_{\mathrm{i}} \boldsymbol{s}_{j}=0
\end{array}\right.
$$

It is worth noting that the cross product in the second line of equation (26) only has two independent constraints, being the third equation linearly dependent on the other two equations. The five scalar constraint equations yield only one relative degree of freedom for this joint, which is a rotation about the common axis of the revolute joint.

A multi-body system is a collection of bodies that is acted upon by forces and moments. These bodies are interconnected to each other by different types of kinematic joints that constrain their relative motion in different forms. For a constrained multibody system, the kinematic joints can be described by a set of algebraic equations as

$$
\boldsymbol{\Phi}(\boldsymbol{q}, t)=\mathbf{0}
$$

where $\boldsymbol{q}$ is the generalized coordinates vector and $t$ is the time variable. Each spherical joint contributes with three equations to the system of equations (27) while each perfect revolute joint contributes with five equations. Differentiating equation (27) with respect to time yields the velocity constraint equations. After a second differentiation with respect to time, the acceleration constraint equations are obtained as

$$
\Phi_{\mathrm{q}} \ddot{\boldsymbol{q}}=\gamma
$$

in which $\boldsymbol{\Phi}_{\mathbf{q}}$ is the Jacobian matrix of the constraint equations, $\ddot{\mathbf{q}}$ the acceleration vector, and $\boldsymbol{\gamma}$ the right-hand side of acceleration equations, which 


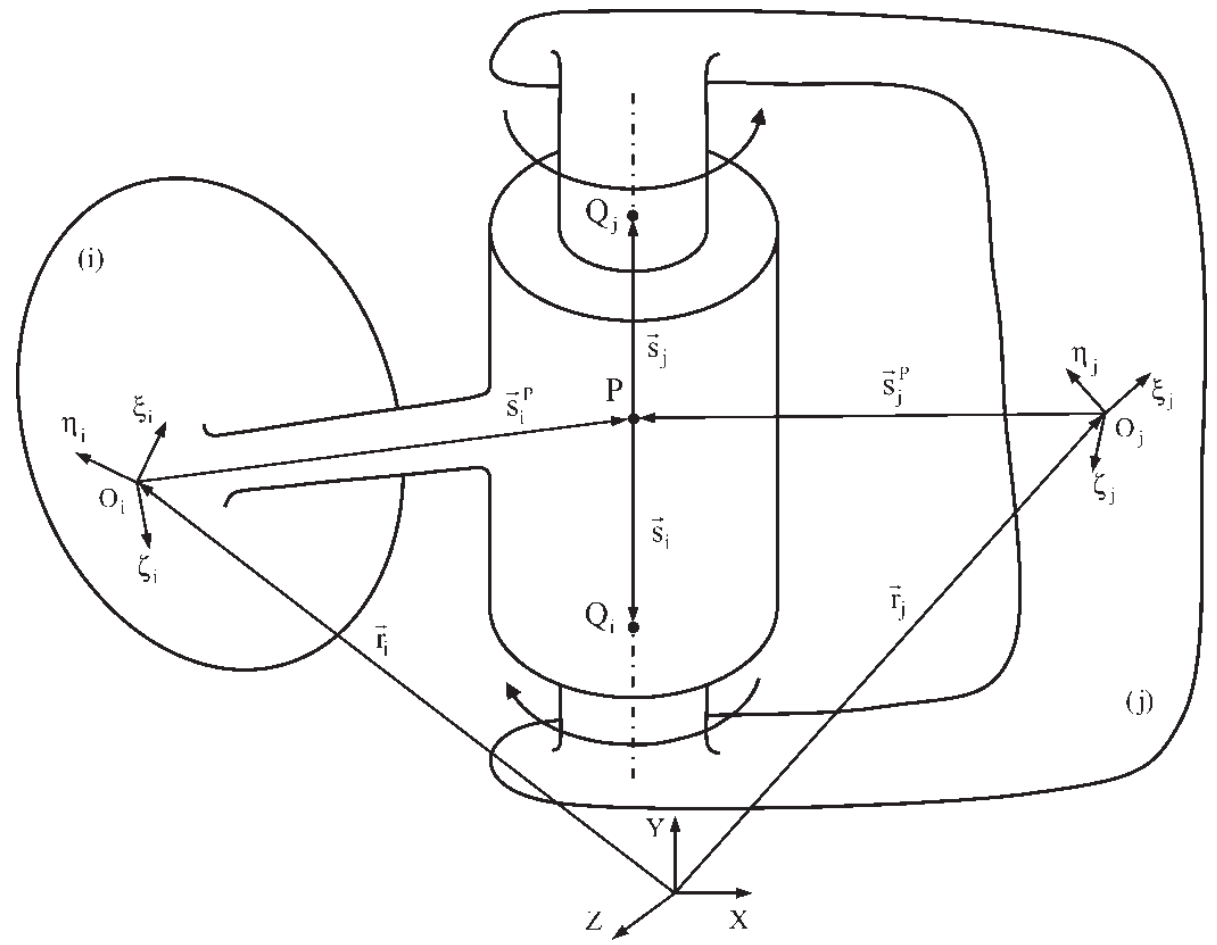

Fig. 11 Perfect spatial revolute joint in a multi-body system

contains the terms that are exclusively function of velocity, position, and time.

The translational and rotational equations of motion for an unconstrained multi-body system of rigid bodies are written as

$$
\mathbf{M} \ddot{\boldsymbol{q}}=\mathbf{g}
$$

where $\mathbf{M}$ is the global system mass matrix, containing the mass and moments of inertia of all bodies, and $\mathbf{g}$ is the generalized force vector that contains all external forces and moments applied on the system.

Using the Lagrange multipliers technique, the constraint equations (27) is added to the equations of motion (29). The equations of motion are written together with the second time derivative of constraint equations (28), yielding a system of equations written as

$$
\left[\begin{array}{cc}
\mathbf{M} & \boldsymbol{\Phi}_{\mathbf{q}}^{\mathrm{T}} \\
\boldsymbol{\Phi}_{\mathbf{q}} & 0
\end{array}\right]\left\{\begin{array}{l}
\ddot{\boldsymbol{q}} \\
\boldsymbol{\lambda}
\end{array}\right\}=\left\{\begin{array}{l}
\boldsymbol{g} \\
\gamma
\end{array}\right\}
$$

where $\boldsymbol{\lambda}$ is the vector of Lagrange multipliers, which physically are related to the joint reaction forces. The reaction forces, owing to the kinematic joints are expressed as [17]

$$
\mathbf{g}^{(\mathbf{c})}=-\boldsymbol{\Phi}_{\mathbf{q}}^{\mathrm{T}} \boldsymbol{\lambda}
$$

Equation (30) is a differential-algebraic equation that has to be solved and the resulting accelerations integrated in time. However, they do not use explicitly the position and velocity constraint equations allowing for a drift in the system constraints to develop. In order to keep under control such constraint violation during the numerical integration the Baumgarte stabilization technique is employed, and equation (30) modified as

$$
\left[\begin{array}{cc}
\mathbf{M} & \boldsymbol{\Phi}_{\mathbf{q}}^{\mathrm{T}} \\
\boldsymbol{\Phi}_{\mathbf{q}} & 0
\end{array}\right]\left\{\begin{array}{l}
\ddot{\boldsymbol{q}} \\
\boldsymbol{\lambda}
\end{array}\right\}=\left\{\begin{array}{c}
\boldsymbol{g} \\
\boldsymbol{\gamma}-2 \alpha \boldsymbol{\Phi}-\beta^{2} \boldsymbol{\Phi}
\end{array}\right\}
$$

where $\alpha$ and $\beta$ are prescribed positive constants that represent the feedback control parameters for the velocities and positions constraint violations $[\mathbf{1 8}$, 19]. According to this formulation, the dynamic response of multi-body systems involves the evaluation of the vectors $\boldsymbol{g}$ and $\boldsymbol{\gamma}$, for each time step. Then, equation (32) is solved for the system accelerations $\ddot{\boldsymbol{q}}$. These accelerations together with the velocities $\dot{\boldsymbol{q}}$ are integrated in order to obtain the new velocities $\dot{\boldsymbol{q}}$ and positions $\boldsymbol{q}$ for the time step. This process is repeated until the complete description of system motion is performed.

At this point, it is worth noticing that perfect joints are introduced in equation (30) or (32) as kinematic constraints. However, imperfect joints, i.e. joints with clearances, are introduced in equation (30) or (32) as forces in their right-hand sides, instead. 


\section{DEMONSTRATIVE APPLICATION TO A DOUBLE PENDULUM}

In order to examine the suitability of the formulation developed to describe the spatial revolute joint clearance, a double pendulum with the configuration shown in Fig. 12 is presented here. The numbering of the bodies of the system and their local coordinate frames are shown in Fig. 12 as well. The double pendulum is made up of three rigid bodies, which are the ground body and two arms. One ideal revolute joint connects the two pendulum arms whereas a spatial revolute joint clearance, with a radial clearance size of $0.5 \mathrm{~mm}$, is in place between the ground and body 2 .

Initially, the double pendulum is resting in the $X Z$ plane position with pendulum arms perpendicular to each other. The system is then released from this initial configuration only subjected to gravity, which is taken as acting in the positive $Y$-direction. The geometric dimensions and inertia properties of the double pendulum are listed in Table 1, whereas the dynamic parameters used in simulations are shown in Table 2.

In order to access the influence of the spatial revolute clearance joint, in the dynamic performance of the double pendulum, some of the kinematic and dynamic characteristics of the system response during the first four seconds of simulation are analysed here, being the results compared to those obtained for a system with ideal joints. The Hertz contact law with hysteresis damping factor, given by equation (24), is used to evaluate the

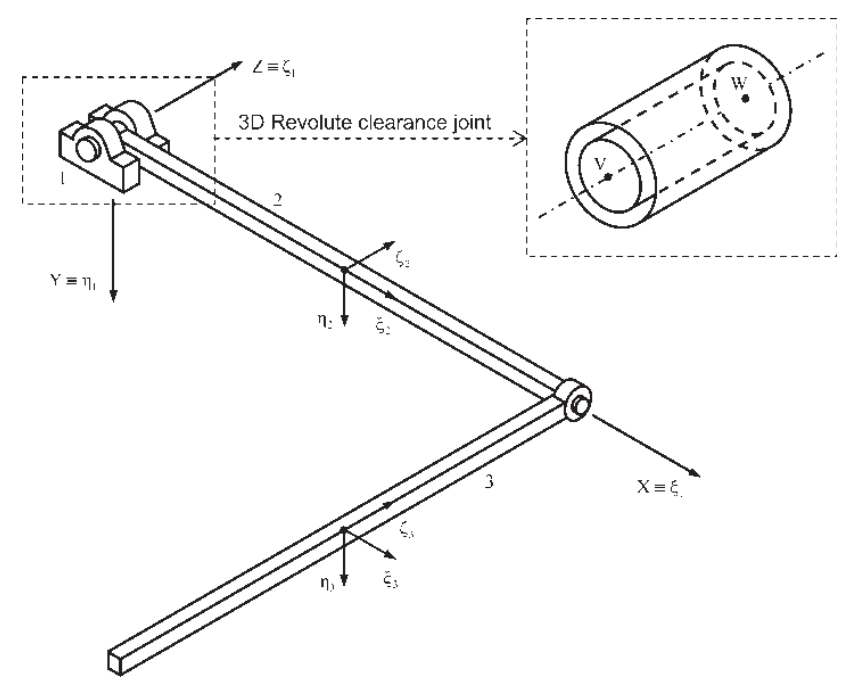

Fig. 12 Double pendulum modelled by two rigid bars and two revolute joints. Each bar is considered as a prismatic homogeneous rigid body with square section of $0.03 \mathrm{~m} \times 0.03 \mathrm{~m}$
Table 1 Governing properties for the double pendulum

\begin{tabular}{llllll}
\hline & & & \multicolumn{3}{c}{ Moment of inertia $\left(\mathrm{Kgm}^{2}\right)$} \\
\cline { 4 - 6 } $\begin{array}{l}\text { Body } \\
\mathrm{nr}\end{array}$ & $\begin{array}{l}\text { Length } \\
(\mathrm{m})\end{array}$ & $\begin{array}{l}\text { Mass } \\
(\mathrm{Kg})\end{array}$ & $I_{\xi \xi}$ & $I_{\eta \eta}$ & $I_{\zeta \zeta}$ \\
\hline 2 & 1.0 & 7.02 & 0.0010 & 0.5855 & 0.5855 \\
3 & 1.0 & 7.02 & 0.5855 & 0.5855 & 0.0010 \\
\hline
\end{tabular}

contact forces caused by the impact in the joint clearance.

Figure 13(a) shows the normal contact force developed in the revolute clearance joint, during the first impact, and the reaction force at the ideal joint. The plotted reaction force is the module of the joint force in $X$-direction developed at the ideal revolute joint that connects the two pendulum arms. In Fig. 13(a), it is observed that the reaction force shape is similar to the shape of the contact force at the clearance joint. The maximum reaction force is about 50 per cent of the contact force. Figure 13(b) shows the hysteresis curves for the first three impacts, developed at the clearance joint. The contact force decreases for each impact, suggesting that some of the system energy is dissipated from impact to impact. This energy dissipated is measured as the area enclosed by the hysteresis plot and is due to the gravitational force being the only external action, as no other external forces or drivers are applied on the system.

The position and acceleration of the centre of mass of body 3 in the $Y$-direction are plotted in Figs 14(a) and 14(b). Since the double pendulum has an open-loop topology, the existence of a joint clearance clearly influences the global position of the bodies of the system. Furthermore, the deviation from the ideal case also results from the fact that the gravitational force and the impact forces at the joint clearance are the only external actions that act on the system. The effect of the impacts, that occur at the joint clearance, produces very high peaks in the different quantities used to represent the system's response of the double pendulum, as it is shown in Fig. 14(b).

The effect of the existence of a revolute joint clearance in the global motion of the double pendulum is illustrated in Fig. 15, in which the trajectory of the centre of mass of the end arm is plotted during the first three seconds of simulation.

Table 2 Simulation parameters for the double pendulum

\begin{tabular}{|c|c|c|c|}
\hline Bearing radius & $10.0 \mathrm{~mm}$ & $\begin{array}{l}\text { Restitution } \\
\text { coefficient }\end{array}$ & 0.9 \\
\hline Journal radius & $9.5 \mathrm{~mm}$ & Young's modulus & $207 \mathrm{GPa}$ \\
\hline Radial clearance & $0.5 \mathrm{~mm}$ & Poisson's ratio & 0.3 \\
\hline
\end{tabular}




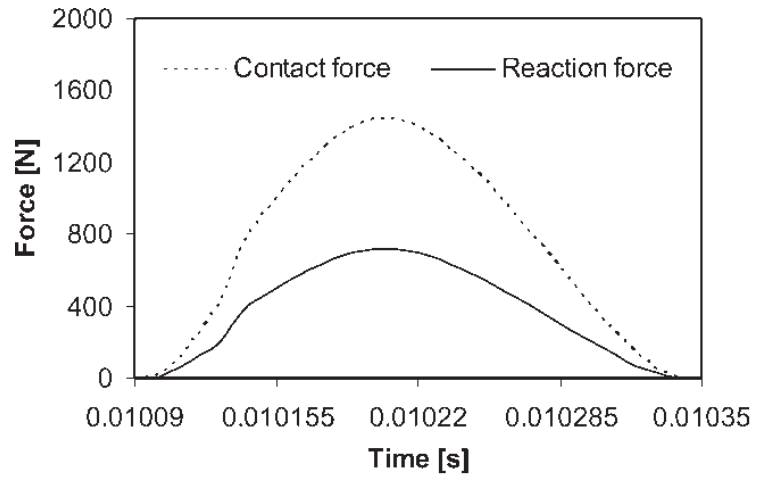

(a)

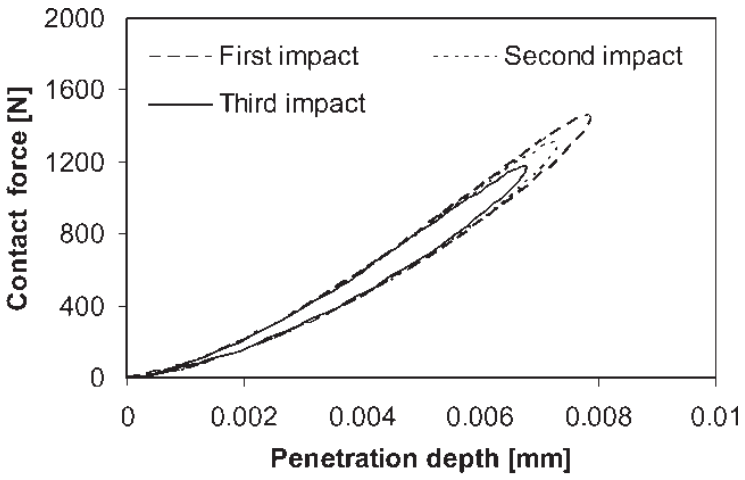

(b)

Fig. 13 (a) Contact force developed at the revolute joint clearance and reaction force in the ideal revolute joint that connects the two pendulum arms; (b) hysteresis loop of the first three impacts at the joint clearance

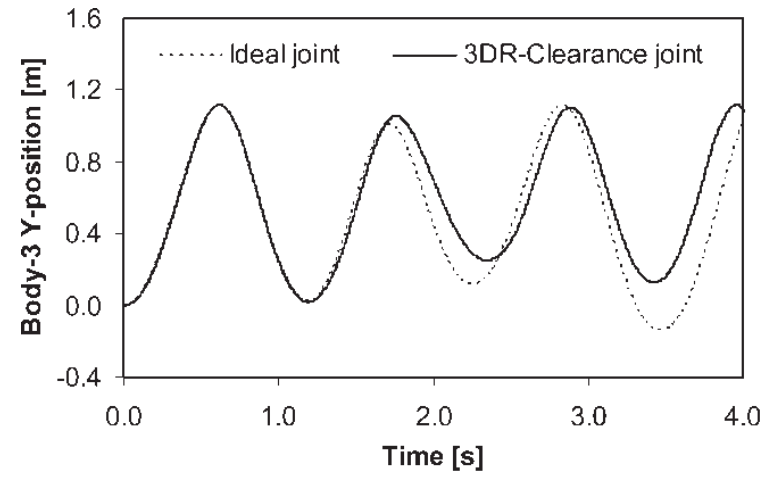

(a)

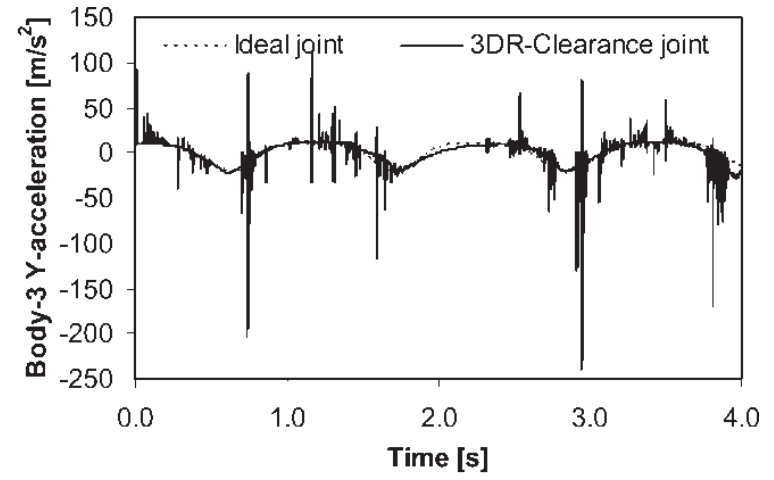

(b)

Fig. 14 (a) $Y$-position of body-3 centre of mass; (b) $Y$-acceleration of body-3 centre of mass

Figure 16 shows the module of the eccentricity vector for both journal bases of the revolute joint clearance, as referred in Fig. 12. It can be observed that, for the first impacts, the trajectories of the two bases are coincident. However, after that, the impacts between the journal and bearing wall take

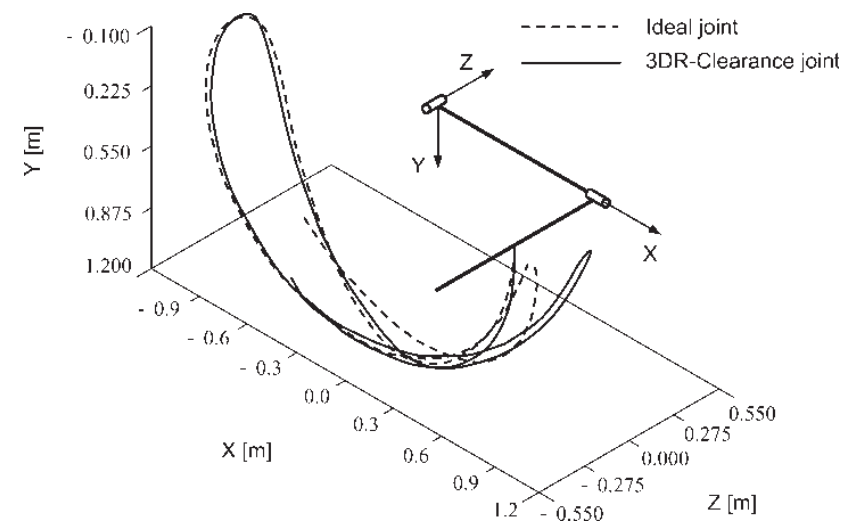

Fig. 15 Trajectory of the centre of mass of the end arm during the first three seconds of simulation place at different instants of time, meaning that some misalignment occurs, and, consequently, only one of the journal bases contacts with the bearing at a given time. This phenomenon can be observed in Fig. 17 where a sequence of frames from a computer animation of the journal trajectory, relative to the

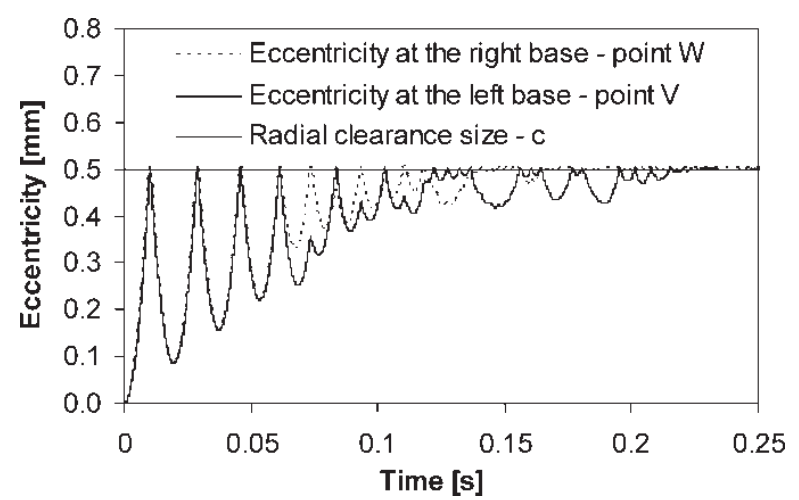

Fig. 16 Module of the eccentricity vector for the two journal bases of the revolute joint clearance 

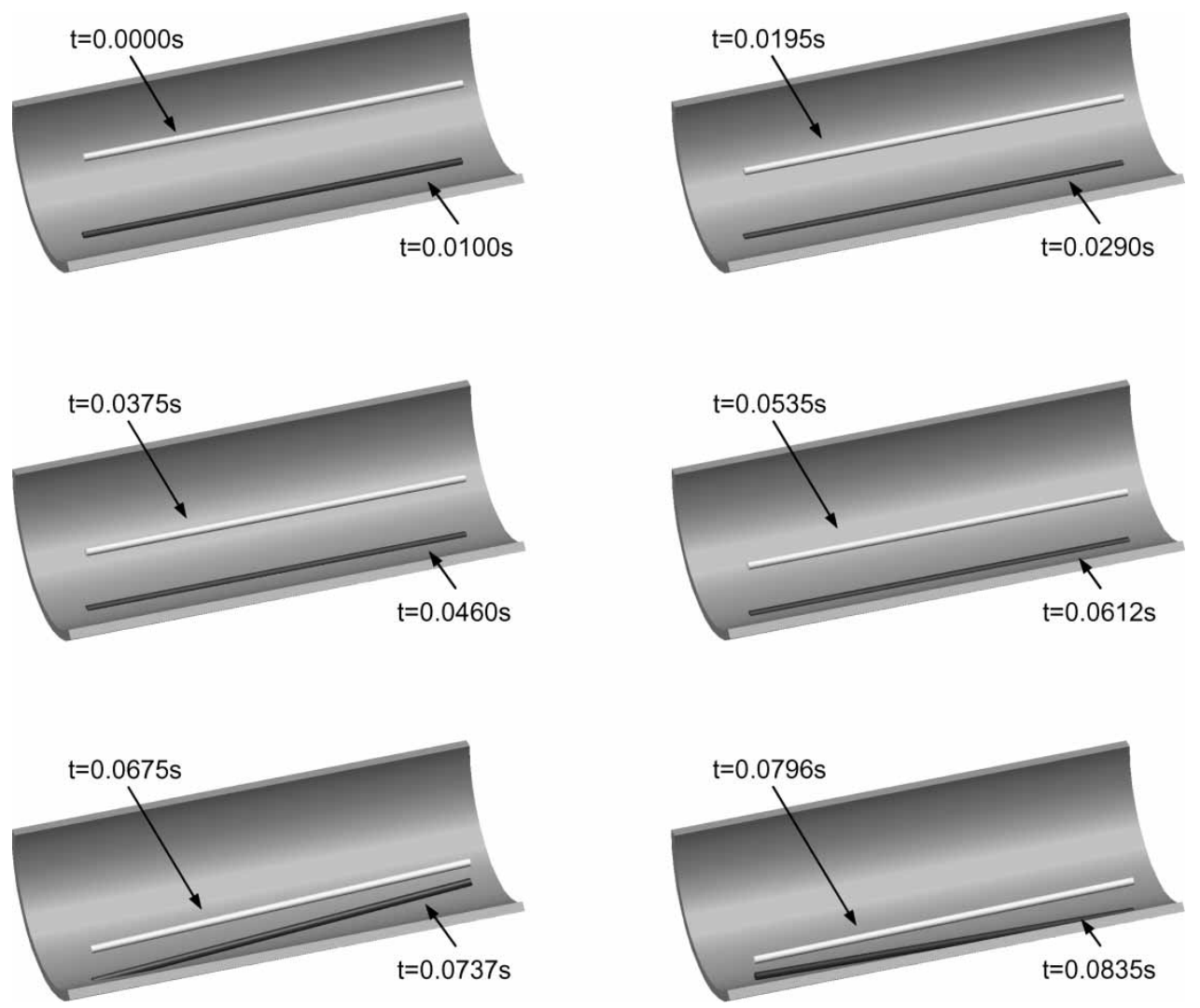

Fig. 17 Sequence of positions representing trajectory of the journal inside the bearing for first instants of simulation. The clearance size is exaggerated for clarity

bearing boundaries, is shown. The contact situations are represented by a dark journal, whereas the noncontact cases are represented by a light journal. In the animation frame sequence, the rebound effect, during the first impacts, is clearly visible. Moreover, the height of the rebound decreases from impact to impact due to the energy loss.

It should be highlighted that the overall results obtained in this work are in line with those of published on this field $[\mathbf{1}, \mathbf{4}]$. In addition, the methodology proposed throughout this paper was extended by Flores et al. [20] in order to quantify the expected wear due to contact-impact phenomenon in clearance joints. Moreover, under the framework of multi-body systems formulation, based on this work, it is also possible to have design guidelines with the intent to define the maximum clearance size that allows a desired level of tolerance on the output variables and to define a criterion that will be helpful in relating the clearance and manufacturing costs. It is well known that in the most machines and mechanisms, the joints operate with some lubricated fluid in order to provide protection against wear by reducing friction, and to introduce some damping to the system and dissipate undesired vibrations [6]. Another important factor that can influence the performance of multi-body systems with impacts is the bodies' elasticity $[\mathbf{9}, \mathbf{1 0}]$. Indeed, some research works $[\mathbf{2 1}, \mathbf{2 2}]$ demonstrated that the level of impact forces due to collisions within clearance joints can be reduced by including the bodies' elasticity in the analysis.

\section{CONCLUSIONS}

In this paper, a general methodology for dynamic characterization of mechanical systems with spatial revolute joints with clearance was formulated, for implementation on a general purpose multi-body computer code. The joint clearance description uses Cartesian coordinates, the joint elements are modelled as impacting bodies, and the dynamics of the joint is controlled by contact-impact forces. These impacts and contacts are described by a continuous contact force model that takes into account the geometric and mechanical characteristics of the contacting bodies. The normal force is evaluated as function of the elastic pseudo-penetration depth between the impacting bodies, coupled with a nonlinear viscous-elastic factor representing the energy dissipation during the impact process. For this 
continuous contact force model, it is assumed that the compliance and damping coefficients are available. The model proposed is useful to predict the peak values of the contact forces and position, velocity and acceleration variations due the presence of the clearance.

A double pendulum was used as a numerical application to illustrate the spatial revolute joint clearance formulation. The overall results presented in this paper show that the introduction of clearance joints in spatial multi-body mechanical systems significantly influences the prediction of components' position and drastically increases the peaks in acceleration at the joints. Moreover, the system's response clearly tends to be non-linear when a joint clearance is included. This is a fundamental feature mainly in high speed and precision mechanisms where the accurate predictions are essential for the systems' design. The methodology can also be used to investigate the effect of parameters variation and to optimize the revolute joints with clearance. Note, however, that the presence of flexibility in the system links or lubrication in the joints can change dramatically the mechanical system behaviour. These other effects can be analysed with more complete models that do not change the way clearances are taken into account.

\section{ACKNOWLEDGEMENTS}

The research work presented in this paper was supported by Fundação para a Ciência e a Tecnologia and partially financed by Fundo Comunitário Europeu FEDER under project POCTI/2001/EME/ 38281, entitled 'Dynamic of Mechanical Systems with Joint Clearances and Imperfections'.

\section{REFERENCES}

1 Dubowsky, S. and Freudenstein, F. Dynamic analysis of mechanical systems with clearances, part 1: formulation of dynamic model. J. Eng. Ind., 1971, 93(1), 305-309.

2 Bahgat, B. M., Osman, M. O. M., and Sankar, T. S. On the effect of bearing clearances in the dynamic analysis of planar mechanisms. J. Mech. Eng. Sci., 1979, 21(6), 429-437.

3 Haines, R. S. Survey: 2-dimensional motion and impact at revolute joints. Mech. Mach. Theory, 1980, 15, 361-370.

4 Ravn, P. A continuous analysis method for planar multibody systems with joint clearance. Multibody Syst. Dyn., 1998, 2, 1-24.

5 Flores, P., Ambrósio, J., and Claro, J. P. Dynamic analysis for planar multibody mechanical systems with lubricated joints. Multibody Syst. Dyn., 2004, 12, $47-74$.
6 Flores, P., Lankarani, H. M., Ambrósio, J., and Claro, J. C. P. Modelling lubricated revolute joints in multi-body mechanical systems. Proc. Instn Mech. Engrs, Part K: J. Multi-body Dynamics, 2004, 218, $183-190$.

7 Lankarani, H. M. and Nikravesh, P. E. A contact force model with hysteresis damping for impact analysis of multibody systems. J. Mech. Des., 1990, 112, 369-376.

8 Haug, E. J., Wu, S. C., and Yang, S. M. Dynamics of mechanical systems with coulomb friction, stiction, impact and constraint addition deletion - I theory. Mech. Mach. Theory, 1986, 21(5), 401-406.

9 Khulief, Y. A. and Shabana, A. A. Dynamic analysis of constrained system of rigid and flexible bodies with intermittent motion. J. Mech. Transm. Autom. Des., 1986, 108, 38-45.

10 Khulief, Y. A. and Shabana, A. A. A continuous force model for the impact analysis of flexible multibody systems. Mech. Mach. Theory, 1987, 22(3), 213-224.

11 Ambrósio, J. Efficient kinematic joint description for flexible multi-body systems experiencing linear and nonlinear deformations. Int. J. Num. Methods Eng., 2003, 56, 1771-1793.

12 Nikravesh, P. E. Computer-aided analysis of mechanical systems, 1988 (Prentice Hall, Englewood Cliffs, New Jersey).

13 Rahnejat, H. Multi-body dynamics: historical evolution and application. Proc. Instn Mech. Engs, Part C: J. Mechanical Engineering Science, 2000, 214(C1), 149-173.

14 Timoshenko, S. P. and Goodier, J. N. Theory of elasticity, 1970 (McGraw Hill, New York).

15 Hertz, H. On the contact of solids - on the contact of rigid elastic solids and on hardness. Miscellaneous papers (Translated by D. E. Jones and G. A. Schott), 1896, pp. 146-183 (MacMillan and Co. Ltd., London).

16 Hunt, K. H. and Crossley, F. R. Coefficient of restitution interpreted as damping in vibroimpact. J. Appl. Mech., 1975, 7, 440-445.

17 García de Jálon, J. and Bayo, E. Kinematic and dynamic simulations of multi-body systems, 1994 (SpringerVerlag, New York).

18 Baumgarte, J. Stabilization of constraints and integrals of motion in dynamical systems. Comput. Methods Appl. Mech. Eng., 1972, 1, 1-16.

19 Flores, P. Dynamic analysis of mechanical systems with imperfect kinematic joints. PhD Dissertation, University of Minho, Guimarães, Portugal, 2005.

20 Flores, P., Claro, J. C. P., Ambrósio, J., and Lankarani, H. M. Modelling and simulation of wear on the dynamics of mechanical systems. 5th International Conference on Mechanics and materials in design, Porto, Portugal, 24-26 July 2006, p. 14.

21 Soong, K. An analytical and experimental study of the elastodynamic response characteristics of planar linkage mechanism with bearing clearances. PhD Dissertation, Michigan State University, Michigan, 1988.

22 Dubowsky, S. and Moening, M. F. An experimental and analytical study of impact forces in elastic mechanical systems with clearances. Mech. Mach. Theory, 1978, 13, 451-465. 


\begin{tabular}{|c|c|c|c|}
\hline \multicolumn{2}{|c|}{ APPENDIX } & $\dot{\delta}^{(-)}$ & initial impact velocity $(\mathrm{m} / \mathrm{s})$ \\
\hline \multirow{2}{*}{\multicolumn{2}{|c|}{ Notation }} & $\Delta$ & increment \\
\hline & & $\xi \eta \zeta$ & three-dimensional body-fixed coordinate \\
\hline A & rotational transformation matrix & & system \\
\hline$c$ & radial clearance $(\mathrm{m})$ & $\theta$ & angular position (rad) \\
\hline$c_{e}$ & coefficient of restitution & $\lambda$ & vector of Lagrange multipliers associated \\
\hline E & Young's modulus of elasticity $\left(\mathrm{N} / \mathrm{m}^{2}\right)$ & & with constraints \\
\hline $\boldsymbol{e}$ & eccentricity vector & $v$ & poisson's ratio \\
\hline$e$ & eccentricity magnitude (m) & $\Phi$ & vector of kinematic constraints \\
\hline$\dot{e}$ & eccentricity or radial velocity $(\mathrm{m} / \mathrm{s})$ & $\Phi_{\mathbf{q}}$ & Jacobian matrix of the kinematic \\
\hline$f$ & force vector & & constraint equations \\
\hline$F_{\mathrm{N}}$ & normal contact force $(\mathrm{N})$ & $\dot{\Phi}$ & constraint velocity equation \\
\hline$g$ & generalized force vector $(\mathrm{N}, \mathrm{Nm})$ & $\Phi$ & constraint acceleration equation \\
\hline$g^{(c)}$ & vector of constraint reaction & ()$^{\mathrm{T}}$ & matrix or vector transpose \\
\hline & equation $(\mathrm{N}, \mathrm{Nm})$ & $\left({ }^{\prime}\right)$ & components of a vector in a body-fixed \\
\hline$K$ & generalized stiffness $\left(\mathrm{N} / \mathrm{m}^{1.5}\right)$ & & coordinate system \\
\hline $\mathbf{M}$ & system mass matrix $\left(\mathrm{kg}, \mathrm{kgm}^{2}\right)$ & $\left(\begin{array}{l}\cdot \\
(-)\end{array}\right.$ & first derivative with respect to time \\
\hline$n$ & normal unit vector & $(")$ & second derivative with respect to time \\
\hline$q$ & vector that contains the state of positions, & (д) & partial derivative \\
\hline & or vector of generalized coordinates & $(\sim)$ & Skew-symmetric matrix or vector \\
\hline$\dot{q}$ & vector that contains the state of velocities & \multirow{2}{*}{\multicolumn{2}{|c|}{ Subscripts }} \\
\hline$\ddot{q}$ & vector that contains the state of & & \\
\hline $\mathrm{R}$ & $\begin{array}{l}\text { accelerations } \\
\text { radius }(\mathrm{m})\end{array}$ & $i$ & relative to body $i$ \\
\hline$r$ & $\begin{array}{l}\text { radius }(\mathrm{m}) \\
\text { global position vector }\end{array}$ & $j$ & relative to body $j$ \\
\hline $\boldsymbol{s}^{\prime}$ & local position vector & $\mathrm{N}$ & normal direction \\
\hline $\mathrm{t}$ & time $(\mathrm{s})$ & $\mathrm{q}$ & generalized coordinate \\
\hline$t$ & tangential unit vector & $\mathrm{r}$ & radial direction \\
\hline \multirow[t]{2}{*}{$X Y Z$} & three-dimensional global coordinate & $\mathrm{T}$ & tangential direction \\
\hline & system & \multicolumn{2}{|c|}{ Superscripts } \\
\hline$\alpha$ & Baumgarte stabilization coefficient & 0 & initial conditions \\
\hline$\beta$ & Baumgarte stabilization coefficient & $\mathrm{P}$ & generic point $\mathrm{P}$ \\
\hline \multirow[t]{3}{*}{$\gamma$} & right-hand side vector of acceleration & $\mathrm{r}$ & revolute joint \\
\hline & equations, or vector of quadratic velocity & $\mathrm{s}$ & spherical joint \\
\hline & terms & $x$ & $X$-direction \\
\hline$\delta$ & penetration depth (m) & $y$ & $Y$-direction \\
\hline$\dot{\delta}$ & penetration velocity $(\mathrm{m} / \mathrm{s})$ & $z$ & $Z$-direction \\
\hline
\end{tabular}

